\title{
Observability Analysis and Optimal Sensor Placement in Stereo Radar Odometry
}

\author{
Andreu Corominas-Murtra, Joan Vallvé, Joan Solà, Ismael Flores and Juan Andrade-Cetto
}

\begin{abstract}
Localization is the key perceptual process closing the loop of autonomous navigation, allowing self-driving vehicles to operate in a deliberate way. To ensure robust localization, autonomous vehicles have to implement redundant estimation processes, ideally independent in terms of the underlying physics behind sensing principles. This paper presents a stereo radar odometry system, which can be used as such a redundant system, complementary to other odometry estimation processes, providing robustness for long-term operability. The presented work is novel with respect to previously published methods in that it contains: (i) a detailed formulation of the Doppler error and its associated uncertainty; (ii) an observability analysis that gives the minimal conditions to infer a $2 \mathrm{D}$ twist from radar readings; and (iii) a numerical analysis for optimal vehicle sensor placement. Experimental results are also detailed that validate the theoretical insights.
\end{abstract}

\section{INTRODUCTION}

Intelligent transportation systems are getting special attention from the mobile robotics community and automotive companies due to safety improvements and potential savings in costs. Beyond self-driving cars, there is a huge amount of applications where autonomous ground vehicles are being developed. Within these applications, transportation of goods and logistics are among the ones offering more economical benefits. The Cargo-ANTs project ${ }^{1}$ aims at developing autonomous ground vehicles (AGV) and autonomous trucks (AT) to operate in a harbor terminal area, 365 days per year, 24 hours per day, loading, unloading and moving shipping containers, being robustness and redundancy two key features to address during developing and engineering phases. In such applications, autonomous navigation is the most critical layer, and localization is the key perceptual process closing the loop, allowing deliberate path following and autonomous operability.

To ensure robust localization, autonomous ground vehicles and trucks have to implement redundant estimation processes, ideally independent in terms of the underlying physics behind sensing principles [7]. Therefore, research on alternative odometry sources beyond the classic wheel encoders is of great importance. Visual based odometry approaches [12], or IMU-visual fusion approaches [3], [10] are not well suited in such scenarios, due to adverse weather and night conditions imposed by full time operations. Lidarbased solutions [5], [11] behave better in night conditions,

Authors are with Institut de Robòtica i Informàtica Industrial (UPCCSIC). Corresponding to: acorominaseiri.upc.edu

This work was supported by the EU Project CargoAnts: Cargo Handling by Automated Next Generation Transportation Systems for Ports and Terminals (FP7-SST-2013-RTD-1-605598).

${ }^{1}$ www.cargo-ants.eu

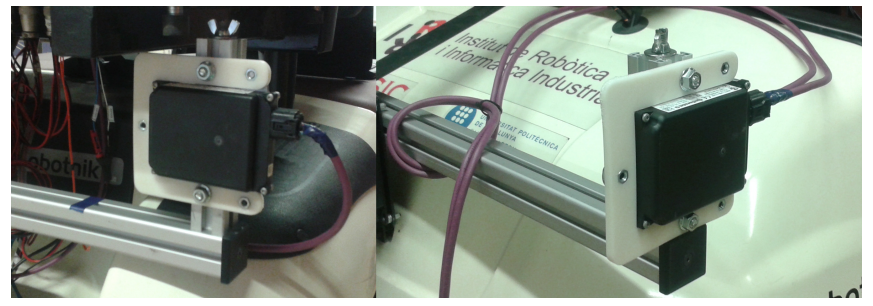

Fig. 1. Stereo radar. Each sensor mounted on a different side of a vehicle.

but still suffer from intense rain or fog. Moreover, lidar devices are often delicate and they could be damaged after several days of intense mechanical vibrations caused by 40 tones-container loading operations. In such context, radar approaches seem to fill the required gap created by these demanding applications.

In autonomous vehicle navigation, radar-based perception systems have been used for collision avoidance [1], mapping [9] and terrain classification [6]. In addition, some authors started to investigate how to compute vehicle ego-motion from the Doppler measurements of the radar echoes [8], [13]. They have recently published promising results using radar devices for odometry estimation in carlike vehicles, using at least a pair of sensors.

Inspired by this recently proposed stereo radar odometry approach (see Figure 1), this paper aims at contributing to the following items:

- Presenting a detailed formulation of the Doppler error and its associated uncertainty.

- Theoretical observability analysis to estimate the 2D twist of a vehicle.

- Numerical analysis for optimal sensor placement onboard the vehicle.

- Providing experimental results validating the theoretical insights presented.

The paper is organized as follows: Section II introduces the problem and the notation used through the paper. Section III is a detailed formulation of the Doppler error and its variance improving the detail given by [8], [13]. Section IV analyses the minimal sensor configuration that observes the full 2D twist (linear velocities and rotation rate), and section $\mathrm{V}$ studies the effects of the radar mounting pose on a platform, while following a criteria of minmum covariance in the output estimate. Finally Section VI presents experimental results, while Section VII concludes the work highlighting the main contributions. 


\section{PROBLEM STATEMENT}

The goal of the presented stereo radar odometry system is to estimate the twist of a vehicle. The twist comprises the linear velocity and the rotation rate with respect to the current vehicle frame, called base frame, $B$. Assuming the vehicle moves in a flat area, corresponding to the $2 \mathrm{D}$ case, this twist state is reduced to:

$$
\mathbf{x}=\left[\begin{array}{ll}
\mathbf{v}^{T} & \omega
\end{array}\right]^{T}=\left[\begin{array}{lll}
v_{x} & v_{y} & \omega
\end{array}\right]^{T}
$$

To estimate this twist from radar measurements, the only sensor readings available are the detections provided by each radar, specifically the azimuth angle, $\alpha_{i j}$, and the Doppler velocity, $u_{i j}$, both for the $i-t h$ device and $j-t h$ detection. Radar devices are mounted on-board the vehicle, each one on a diferent calibrated pose. That is, the $i-t h$ device is located at frame $S_{i}$ with respect to the base frame, i.e, at the mounting point $\mathbf{m}_{i}=\left(m_{i x}, m_{i y}\right)$, with orientation $\beta_{i}$ with respect to the vehicle frontal axis. Figure 2 shows the involved frames and vectors used through the paper.

Let us define the Doppler error

$$
e_{i j}=u_{i j}-\hat{u}_{i j}
$$

as the difference between measured Doppler velocity for sensor $i$ and detection $j, u_{i j}$, and the expected one, $\hat{u}_{i j}$, as well the variance of such error $\sigma_{e_{i j}}^{2}$. Then, the optimal twist that best explains all measurements is given by minimizing a non-linear least squares problem such as:

$$
\left(\mathbf{v}^{*}, \omega^{*}\right)=\underset{(\mathbf{v}, \omega)}{\arg \min }\left(\sum_{i j} \frac{e_{i j}^{2}}{\sigma_{e_{i j}}^{2}}\right)
$$

which we will show in the following to be an optimization problem on the twist variables $v_{x}, v_{y}, \omega$, since both terms $e_{i j}^{2}$ and $\sigma_{e_{i j}}^{2}$ depend on the twist. The purpose of the next section is to detail the computation of both $\hat{u}_{i j}$ and $\sigma_{e_{i j}}^{2}$.

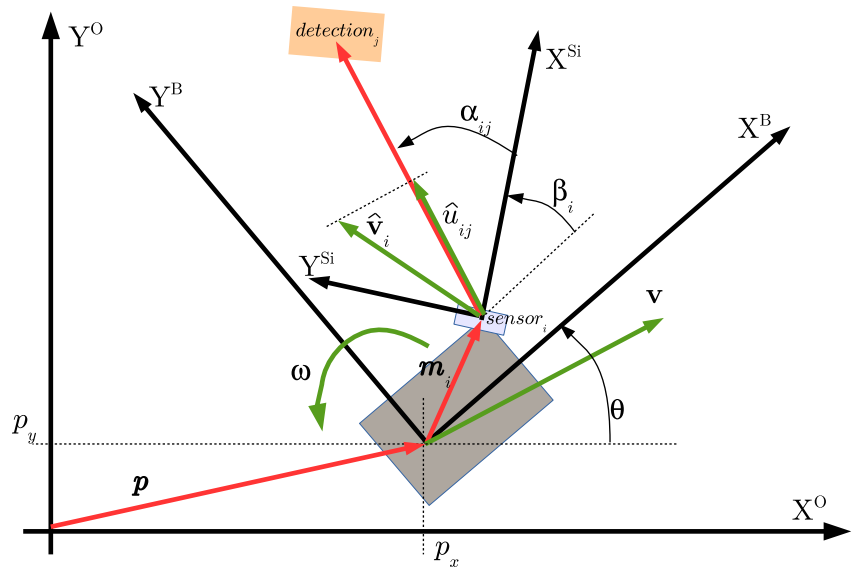

Fig. 2. In black, odometry $(O)$, base $(B)$ and sensor $\left(S_{i}\right)$ frames. Vectors and angles of interest through the paper are also shown.

\section{EXPECTED DOPPLER AND ERROR VARIANCE}

\section{A. Expected Doppler}

The goal of this subsection is to express the expected Doppler velocity, $\hat{u}_{i j}$, for each sensor $i$ and detection $j$, with respect to the vehicle twist $\left(v_{x}, v_{y}, \omega\right)$, sensor mounting parameters $\left(m_{i x}, m_{i y}, \beta_{i}\right)$ and the detection azimuth $\alpha_{i j}$. This constitutes our odometry observer. It's good to point out that this observer does not require any data association neither with previous measurements, nor with any landmark of a map.

Relying on Figure 2, and assuming static objects, the expected Doppler, $\hat{u}_{i j}$, is the projection of the expected radar device linear velocity, $\hat{\mathbf{v}}_{i}$, into the direction given by the azimuth $\alpha_{i j}$ of the $j-t h$ detection from the $i-t h$ radar:

$$
\hat{u}_{i j}=\hat{\mathbf{v}}_{i}^{T}\left[\begin{array}{l}
\cos \alpha_{i j} \\
\sin \alpha_{i j}
\end{array}\right]
$$

In the general 3D case, the term $\hat{\mathbf{v}}_{i}$ is related to the vehicle twist $(\mathbf{v}, \omega)$ as follows:

$$
\hat{\mathbf{v}}_{i}=\mathbf{R}_{B}^{S_{i}}\left(\mathbf{v}+\omega \times \mathbf{m}_{i}\right),
$$

which in $2 \mathrm{D}$ case reduces to:

$$
\hat{\mathbf{v}}_{i}=\left[\begin{array}{cc}
\cos \beta_{i} & -\sin \beta_{i} \\
\sin \beta_{i} & \cos \beta_{i}
\end{array}\right]\left[\begin{array}{l}
v_{x}-\omega m_{i y} \\
v_{y}+\omega m_{i x}
\end{array}\right]
$$

Equation 5 mainly involves two addends, the first relates to the vehicle's linear velocity and the later to the vehicle's rotation rate. Moreover, a rotation matrix is involved, $\mathbf{R}_{B}^{S_{i}}$, to move the resulting velocity to each sensor frame where the Doppler measurements are taken. After some math manipulation of Equations 4 and 6, the expected Doppler is finally expressed as:

$$
\hat{u}_{i j}=\left(v_{x}-\omega m_{i y}\right) \cos \left(\beta_{i}+\alpha_{i j}\right)+\left(v_{y}+\omega m_{i x}\right) \sin \left(\beta_{i}+\alpha_{i j}\right)
$$

which agrees with the expression published in [13].

\section{B. Estimated Error Variance}

The variance of the error factor $e_{i j}$ comes from two sources: the Doppler measurement itself, $u_{i j}$ and the expression of the expected Doppler, $\hat{u}_{i j}$, which also involves the measurement $\alpha_{i j}$, being the azimuth of detection $j$ from radar $i$ (see Equation 7). So the variance associated to $e_{i j}$ is expressed as the sum of two terms:

$$
\sigma_{e_{i j}}^{2}=\left(\frac{\partial e_{i j}}{\partial \alpha_{i j}}\right)^{2} \sigma_{\alpha}^{2}+\sigma_{u}^{2}
$$

where the partial derivative of the error $e_{i j}$ with respect to the azimuth measurement, $\alpha_{i j}$, is:

$\frac{\partial e_{i j}}{\partial \alpha_{i j}}=-\left(v_{x}-\omega m_{i y}\right) \sin \left(\beta_{i}+\alpha_{i j}\right)+\left(v_{y}+\omega m_{i x}\right) \cos \left(\beta_{i}+\alpha_{i j}\right)$

and $\sigma_{\alpha}$ and $\sigma_{u}$ are the standard deviations of azimuth and Doppler respectively, as they are usually specified by the radar device datasheet. 


\section{OBSERVABILITY ANALYSIS}

This section demonstrates which are the conditions to fully observe a 2D twist with the proposed odometry approach. The minimal set-up was already suggested by [8], without showing explicitly the underlying maths behind it.

Equation 7 describes the observation model of the expected Doppler velocity. Given this expression, we compute the Jacobian of the expected error (Equation 2) with respect to the state (2D twist), and assume a minimal set of radar detections. To solve for three degrees of freedom $\left(v_{x}, v_{y}, \omega\right)$ we require, at least, three radar detections.

\section{A. Single Radar Case}

In case all three detections come from a single radar device, the involved Jacobian $\left[\partial v_{x} \partial v_{y} \partial \omega\right]$ is:

$$
\mathbf{J}_{1,3}=\left[\begin{array}{lll}
\cos \varphi_{11} & \sin \varphi_{11} & -m_{1 y} \cos \varphi_{11}+m_{1 x} \sin \varphi_{11} \\
\cos \varphi_{12} & \sin \varphi_{12} & -m_{1 y} \cos \varphi_{12}+m_{1 x} \sin \varphi_{12} \\
\cos \varphi_{13} & \sin \varphi_{13} & -m_{1 y} \cos \varphi_{13}+m_{1 x} \sin \varphi_{13}
\end{array}\right],
$$

where $\varphi_{i j}=\beta_{i}+\alpha_{i j}$, and $\mathbf{J}_{1,3}$ indicates Jacobian for one radar and three detections case. This Jacobian is rank deficient because the third column is a linear combination of the first and second ones. Therefore, the 2D twist cannot be observed with a single radar device. Moreover, it is obvious that adding extra detections does not unlock the rank-deficient situation.

\section{B. Stereo Radar Case}

Therefore, we have to analyse the case with two devices and three detections. In that case, it is useful to redefine a new frame convention to better analyse this situation. Figure 3 shows this useful convention with axis $X^{\prime}$ and $Y^{\prime}$, and parameters $b$ and $\varphi_{i j}^{\prime}$. With this new frame convention, the involved Jacobian leads to the following expression:

$$
\mathbf{J}_{2,3}=\left[\begin{array}{ccc}
\cos \varphi_{11}^{\prime} & \sin \varphi_{11}^{\prime} & -\frac{b}{2} \cos \varphi_{11}^{\prime} \\
\cos \varphi_{12}^{\prime} & \sin \varphi_{12}^{\prime} & -\frac{b}{2} \cos \varphi_{12}^{\prime} \\
\cos \varphi_{21}^{\prime} & \sin \varphi_{21}^{\prime} & \frac{b}{2} \cos \varphi_{21}^{\prime}
\end{array}\right],
$$

which is a full rank matrix exceptuating some special configurations, where the determinant is null, and the 2D twist cannot be observed. These degenerate cases fulfill:

$$
\cos \varphi_{21}^{\prime}\left(\cos \varphi_{11}^{\prime} \sin \varphi_{12}^{\prime}-\sin \varphi_{11}^{\prime} \cos \varphi_{12}^{\prime}\right)=0
$$

which result on the following cases:

- $\varphi_{11}^{\prime}=\varphi_{12}^{\prime}+k \pi, k \in \mathbb{Z}$.

- $\varphi_{21}^{\prime}=\frac{\pi}{2}+k \pi, k \in \mathbb{Z}$.

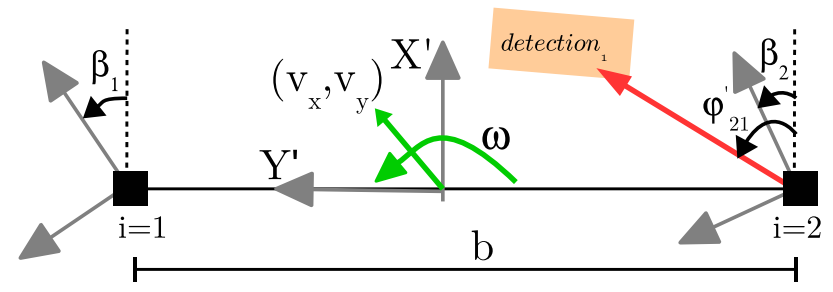

Fig. 3. Frame convention useful to analyse observability in stereo radar.
The first case appears when a single device $i$ detects two objects aligned along the same line of sight. The later case is when the device reporting a single detection senses it aligned with the baseline. In practical situations, due to large amount of radar detections available, these two null-rank cases are rare. They are single configurations among an infinite set of other random situations that could occur in a vehicle moving context. However, a more practical implication arises in RANSAC-based outlier rejection approaches [8], since such null-rank configurations have to be avoided when chosing a minimal subset to solve the problem.

\section{OPTIMAL SENSOR PLACEMENT}

This section provides a numerical analysis to study the optimal sensor placement for radar stereo odometry purpose. The criteria used is to maximize the determinant of the information matrix, while minimal assumptions are considered in order to present a valid methodolgy and illustrative results. This maximization implies a minimization of the estimated uncertainty in the whole twist space [4]. The information matrix over all measurements, $\Lambda$, is defined as:

$$
\Lambda=\sum_{i j} \mathbf{J}_{i j}^{T} \frac{1}{\sigma_{e_{i j}}^{2}} \mathbf{J}_{i j}
$$

where, in our case, $\mathbf{J}_{i j}$ is the Jacobian of a single measurement with respect to the vehicle twist $\left(v_{x}, v_{y}, \omega\right), \sigma_{e_{i j}}^{2}$ is the variance of the Doppler error (see Equation 8), and $\Lambda$ is the resulting $3 \times 3$ information matrix. $\Lambda$ matrix is the inverse of the covariance matrix. Therefore, Equation 13 acts basically as a transformation of inverse uncertainties, from the Doppler space to the twist one.

The determinant of $\Lambda,|\Lambda|$, is the quantity of interest to be maximized, which indicates minimum volume of the covariance ellipsoid in the twist space. This determinant depends on the vehicle twist, the sensor mounting poses and the azimuth of the Doppler detections, but we are specifically interested in the sensor placement dependency. Assuming we find detections equally distributed around the vehicle, we compute $|\Lambda|$ considering $i j$ detections uniformly distributed over all the azimuth aperture for each radar, so azimuth dependence can be removed. This assumption could be also derived according to some environment model, which could reshape the final result. However, the presented methodolgy herein remains valid. Given two radar devices, mounting pose on-board a car-like vehicle requires 6 parameters. We make a second assumption here, which is that sensors are placed on the platform perimeter. In order to be able to plot results in an illustrative way, so we remap mounting poses alongside the vehicle perimeter to a single continuous value, $\eta \in[0,8)$, in the way drawn in Figure 4, being $L, W$ and $D$ the vehicle length, width and distance from base frame to rear side respectively. In that point, given a twist we are able to numerically evaluate $|\Lambda|$ for each pair of mounting poses $\left(\eta_{1}, \eta_{2}\right)$.

In this section, we provide results for our experimental Ackerman platform, with dimensions $L=212 \mathrm{~cm}, W=$ $102 \mathrm{~cm}$ and $D=32 \mathrm{~cm}$. 

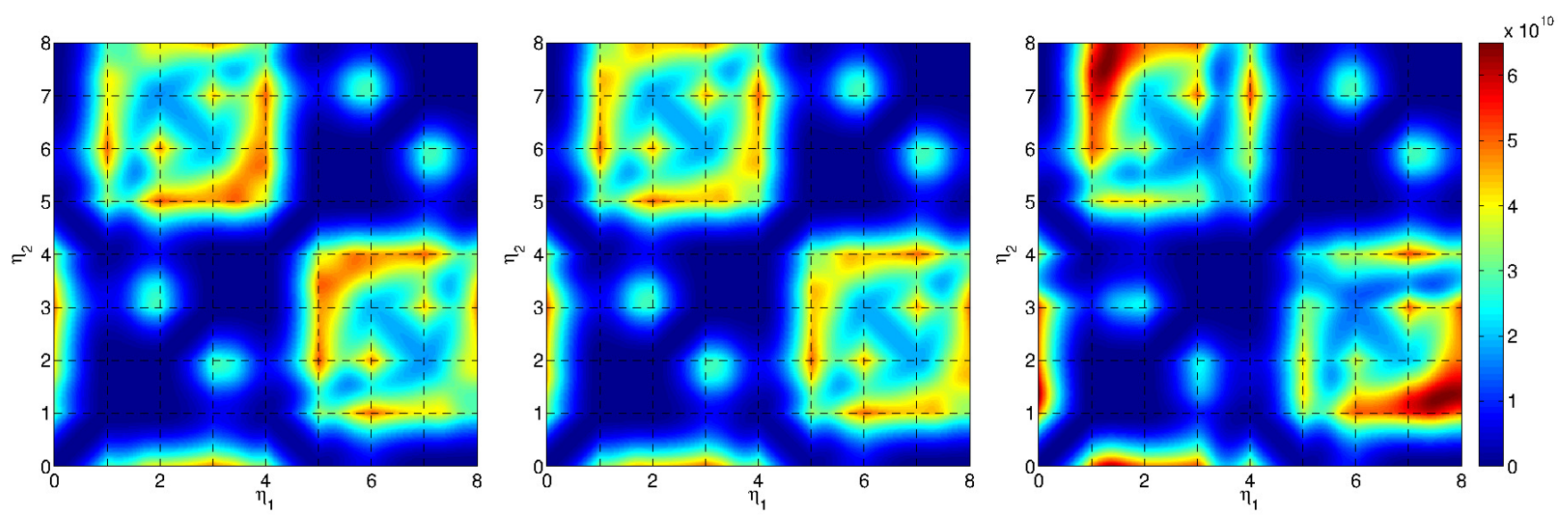

Fig. 5. Value of $|\Lambda|$ for $v_{x}=1.2 \mathrm{~m} / \mathrm{s}, v_{y}=0 \mathrm{~m} / \mathrm{s}$ and $\omega=0.1 \mathrm{rad} / \mathrm{s}$ (left), $\omega=0 \mathrm{rad} / \mathrm{s}$ (centre) and $\omega=-0.3 \mathrm{rad} / \mathrm{s}$ (right).

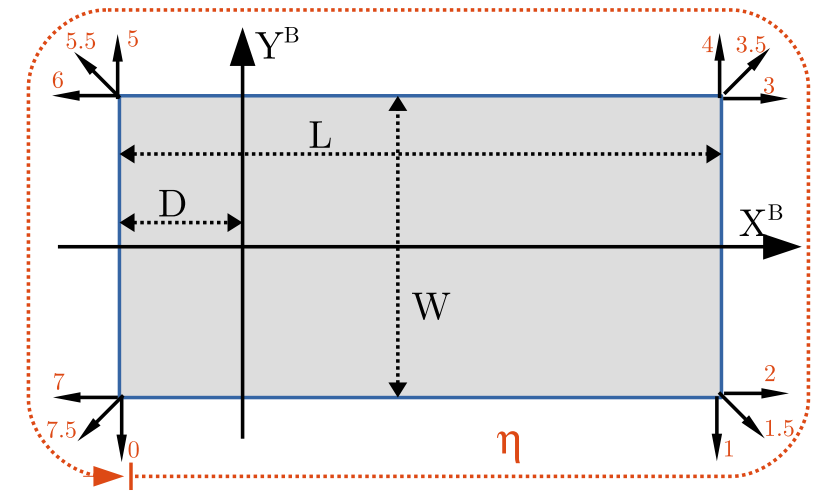

Fig. 4. Mapping between sensor mounting pose on the vehicle's perimeter (small black arrows) and $\eta$ value. This mapping is used in Figures 5 and 6 . Vehicle dimensions length, $L$, width, $W$, and distance $D$ are also indicated.

Figure 5 shows three plots of $|\Lambda|$ value (colormap) according to the mounting pose of each radar $\left(\eta_{1}, \eta_{2}\right)$. The three figures were computed given three diferent motions of the vehicle: soft turn left $\left(v_{x}=1.2 \mathrm{~m} / \mathrm{s}, v_{y}=0 \mathrm{~m} / \mathrm{s}, \omega=\right.$ $0.1 \mathrm{rad} / \mathrm{s}$, left $)$, straight line $\left(v_{x}=1.2 \mathrm{~m} / \mathrm{s}, v_{y}=0 \mathrm{~m} / \mathrm{s}, \omega=\right.$ $0 \mathrm{rad} / \mathrm{s}$, center) and closer turn right $\left(v_{x}=1.2 \mathrm{~m} / \mathrm{s}, v_{y}=\right.$ $0 \mathrm{~m} / \mathrm{s}, \omega=-0.3 \mathrm{rad} / \mathrm{s}$, right). Due to the remapping used to represent sensor mounting poses, the upper-left and lowerright triangles of these plots show the same values, which indicates that switching radar mounting poses between them has no effects. Moreover, the diagonal of such plots means that both sensors are placed at the same pose, so configurations close to that region are poor in terms of odometry estimate. The plot also shows how preferred configurations pair one pose in the vehicle front with one pose in the vehicle rear, so baseline between sensors is longer (upperleft or lower right quarters). A standard configuration in automotive [13], usually conditioned by the collision avoidance system, mounts both devices pointing forward in front of the vehicle, $\eta_{1}=2, \eta_{2}=3$, which is far from being an optimal placement for stereo radar odometry, even if it features a slight local maxima in all three motion cases.
Figure 6 draws the minimum value of $|\Lambda|$ over a set of different motions that our vehicle is able to drive, according to its Ackerman kinematics: $v_{x}=1.2 \mathrm{~m} / \mathrm{s}, v_{y}=0 \mathrm{~m} / \mathrm{s}, \omega \in$ $[-0.3,0.3] \mathrm{rad} / \mathrm{s}$. This plot shows that sensor configuration indicated by $\eta$ values $(1,6)$ is the one featuring the maximum minimum, so it indicates an optimal sensor placement to minimize final twist covariance estimate. Configuration in $\eta$ pair of $(4,7)$ reach the same maximum minimum, since it indicates a symmetrical set-up on vehicle mounting poses (in the $X^{B}$ axis). With respect to the vehicle $Y^{B}$ axis, sensor placement is not symmetric due to the Ackerman kinematics, since the rotation point is centered in the rear axis. Sensor placement pair $(5,2)$ is an instance of such asymmetry, since the peak appearing in the plot is lower than the one in $(1,6)$.

Even if the numerical results are provided for ideal Ackerman motion $\left(v_{y}=0\right)$, it's worth to recall that the proposed approach observes the full 2D twist, since observability conditions studied in section IV are still satisfied. Thus, skidding situations of the platform will be observed.

\section{EXPERIMENTAL RESULTS}

Experimental sessions were carried out with our electric Ackerman car in the Barcelona Robot Lab, an university campus outdoor environment. The vehicle run at linear speeds around $1.2 \mathrm{~m} / \mathrm{s}$ and rotation rates up to $0.3 \mathrm{rad} / \mathrm{s}$. The radar devices used were two Continental SRR20x, operating at $24 \mathrm{GHz}$ in cluster mode. For ground truth purposes, the vehicle was also equipped with wheel encoders and an industrial-grade IMU.

The implemented solution was coded in $\mathrm{C}++$, using the Ceres library [2] as the optimization engine to solve equation 3. A straightforward outlier rejection was implemented based on the residuals obtained after a first optimization call, as well as using a Cauchy loss function. The stereo radar odometry estimate ouput was computed at $5 \mathrm{~Hz}$, so each iteration considered all detections read during the last $200 \mathrm{~ms}$ interval, and used the previous solution as an initial guess.

Several datasets were recorded, and results are presented in two subsections: VI-A, quantitative results in the twist space, and VI-B, qualitative results in the integrated pose. 


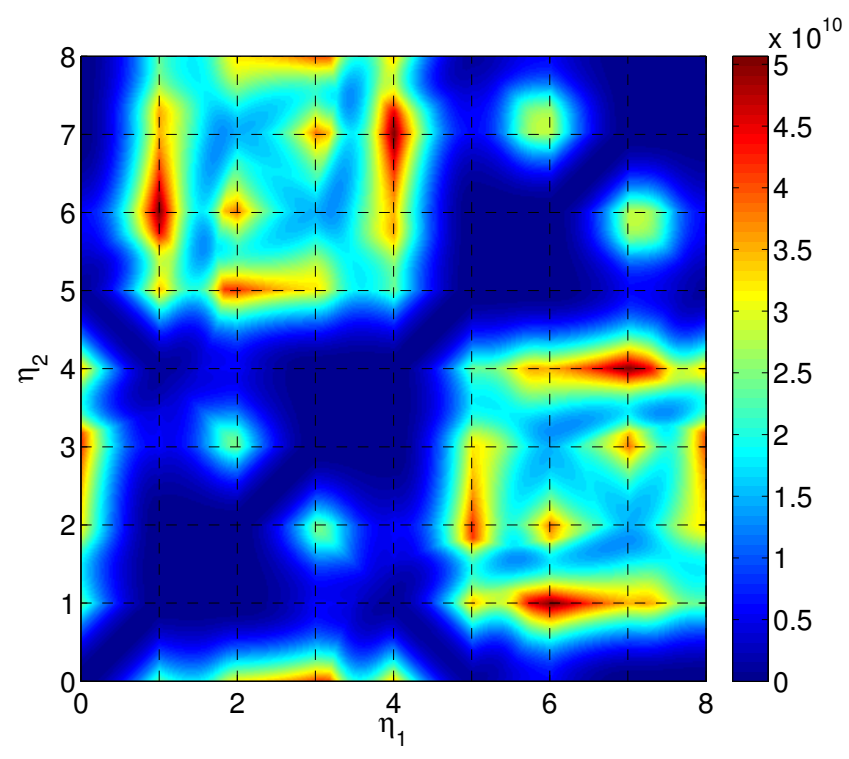

Fig. 6. Minimum value of $|\Lambda|$ over a set of different motions the vehicle is able to drive: $\left\{v_{x}=1.2 \mathrm{~m} / \mathrm{s}, v_{y}=0 \mathrm{~m} / \mathrm{s}, \omega=\{-0.3 \ldots 0.3 \mathrm{rad} / \mathrm{s}\}\right.$

\section{A. Twist Space}

A quantitative validation of the approach, as well as the sensor placement relevance, is provided performing the following experiment. We run with the car around a zerolike path of around $70 \mathrm{~m}$. For an optimal configuration (CFG-A) $\eta_{1}=4, \eta_{2}=7$, we did 5 trials. This configuration corresponds to one device mounted in the front-left corner, pointing sideways, and the second one mounted on the rearright corner, pointing backwards. We then repeated these 5 trials for a non-optimal configuration (CFG-B) $\eta_{1}=$ $3.5, \eta_{2}=1.5$, which corresponds to mounting both radars in the front side, with angles $\beta_{1}=45^{\circ}$ and $\beta_{2}=-45^{\circ}$. For the $v_{x}$ component of the twist we compare the stereo radar estimate against wheel encoders forward velocity. For the $v_{y}$ component, the ground truth is set to be zero, assuming that the vehicle didn't slip while running. For the $\omega$ component of the estimated twist, results are compared with the vertical component of the rotational rate sensed by IMU (substracting a bias estimated from an initial stopped period of 5 seconds for each trial). All results are presented in table I, where error mean and covariance are provided, over the 5 trials. The table also details these mean and covariance values for the straight and turning parts of the zero-like trajectory.

Table I shows that for the $v_{x}$ component, both sensor configurations perform similarly, in both terms of error mean and error covariance. However, for lateral velocity component, $v_{y}$, CFG-A performs much better in terms of covariance, while the mean error is higher than CFG-B, probably due to some unmodelled bias, or due to the fact that the actual ground truth was not $v_{y}=0$, specially when turning. Is in the rotational rate component, $\omega$, where CFGA outperforms cleraly CFG-B, in both the mean and the covariance. This later result, specifically the lower error covariance, is of major interest, since rotational rate is the
TABLE I

TWIST SPACE ERRORS FOR BOTH CONFIGURATIONS IN ZERO-LIKE TRAJECTORIES

\begin{tabular}{|c|c|c|c|c|c|c|}
\hline \multicolumn{7}{|c|}{ Error in $v_{x} ; \mu[\mathrm{m} / \mathrm{s}], \sigma^{2}\left[\mathrm{~m}^{2} / \mathrm{s}^{2}\right]$} \\
\hline & \multicolumn{2}{|c|}{ Total } & \multicolumn{2}{|c|}{ Straight } & \multicolumn{2}{|c|}{ Turning } \\
\hline & $\mu$ & $\sigma^{2}$ & $\mu$ & $\sigma^{2}$ & $\mu$ & $\sigma^{2}$ \\
\hline CFG-A & -0.0170 & 0.0005 & -0.0142 & 0.0005 & -0.0243 & 0.0002 \\
\hline CFG-B & -0.0155 & 0.0004 & -0.0139 & 0.0004 & -0.0189 & 0.0002 \\
\hline \multicolumn{7}{|c|}{ Error in $v_{y} ; \mu[\mathrm{m} / \mathrm{s}], \sigma^{2}\left[\mathrm{~m}^{2} / \mathrm{s}^{2}\right]$} \\
\hline & \multicolumn{2}{|c|}{ Total } & \multicolumn{2}{|c|}{ Straight } & \multicolumn{2}{|c|}{ Turning } \\
\hline & $\mu$ & $\sigma^{2}$ & $\mu$ & $\sigma^{2}$ & $\mu$ & $\sigma^{2}$ \\
\hline CFG-A & -0.0190 & 0.0004 & -0.0156 & 0.0004 & -0.0275 & 0.0004 \\
\hline $\begin{array}{c}\text { CFG-B } \\
\end{array}$ & -0.0050 & 0.0024 & -0.0001 & 0.0022 & -0.0153 & 0.0026 \\
\hline \multicolumn{7}{|c|}{ Error in $\omega ; \mu[\mathrm{rad} / \mathrm{s}], \sigma^{2}\left[\mathrm{rad}^{2} / \mathrm{s}^{2}\right]$} \\
\hline & \multicolumn{2}{|c|}{ Total } & \multicolumn{2}{|c|}{ Straight } & \multicolumn{2}{|c|}{ Turning } \\
\hline & $\mu$ & $\sigma^{2}$ & $\mu$ & $\sigma^{2}$ & $\mu$ & $\sigma^{2}$ \\
\hline CFG-A & 0.0024 & 0.0002 & 0.0030 & 0.0001 & 0.0006 & 0.0003 \\
\hline $\begin{array}{l}\text { CFG-B } \\
\end{array}$ & -0.0031 & 0.0009 & -0.0033 & 0.0008 & -0.0028 & 0.0011 \\
\hline
\end{tabular}

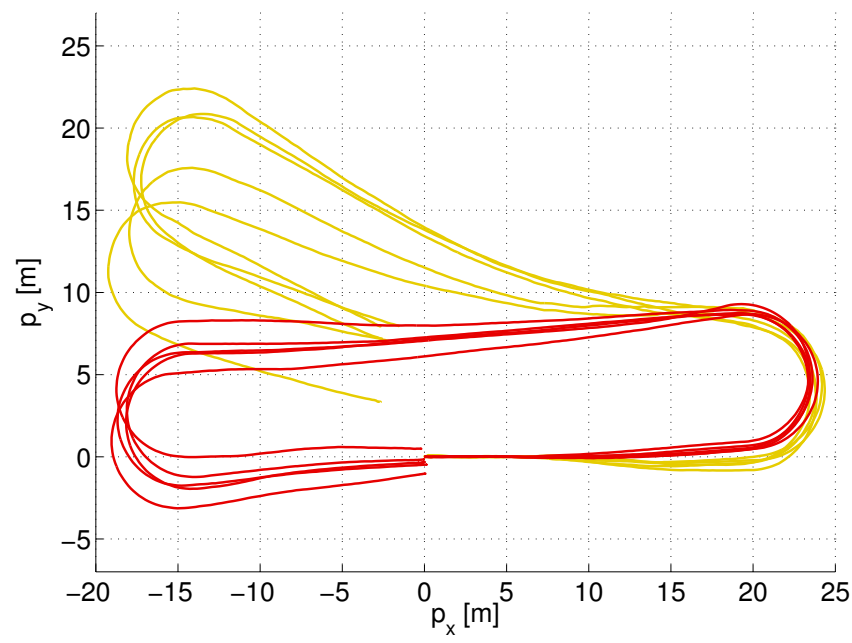

Fig. 7. Integrated pose for optimal sensor placement (CFG-A, red), and non-optimal one (CFG-B, yellow), for the zero-like trajectories

critical component to be estimated by an odometry observer. For instance, odometry based on wheel encoders usually leads to inaccurate and noisy estimation of $\omega$.

\section{B. Integrated Pose}

This subsection shows results in a more qualitative way. Figure 7 plots the integrated poses of the zero-like trajectories for the experiment presented in VI-A. Plots draw integrated pose paths for CFG-A and CFG-B. These plots show qualitatively how the optimal configuration reduces the final error of the integrated pose, thanks to a more accurate estimation of the twist, specially the $\omega$ component.

A second qualitative result was obtained after following an eight-like path of more than $300 \mathrm{~m}$. One trial was done with the optimal configuration of the radars (CFG-A), and a second one with the non-optimal placement (CFGB). Integrated pose trajectories of both trials have been overprinted on a Goolge Maps snapshot of the experimental area (see Figure 8).

In this long eight-like trajectory, the vehicle traversed two short ramps, causing to pitch it, so leading to a rotational 


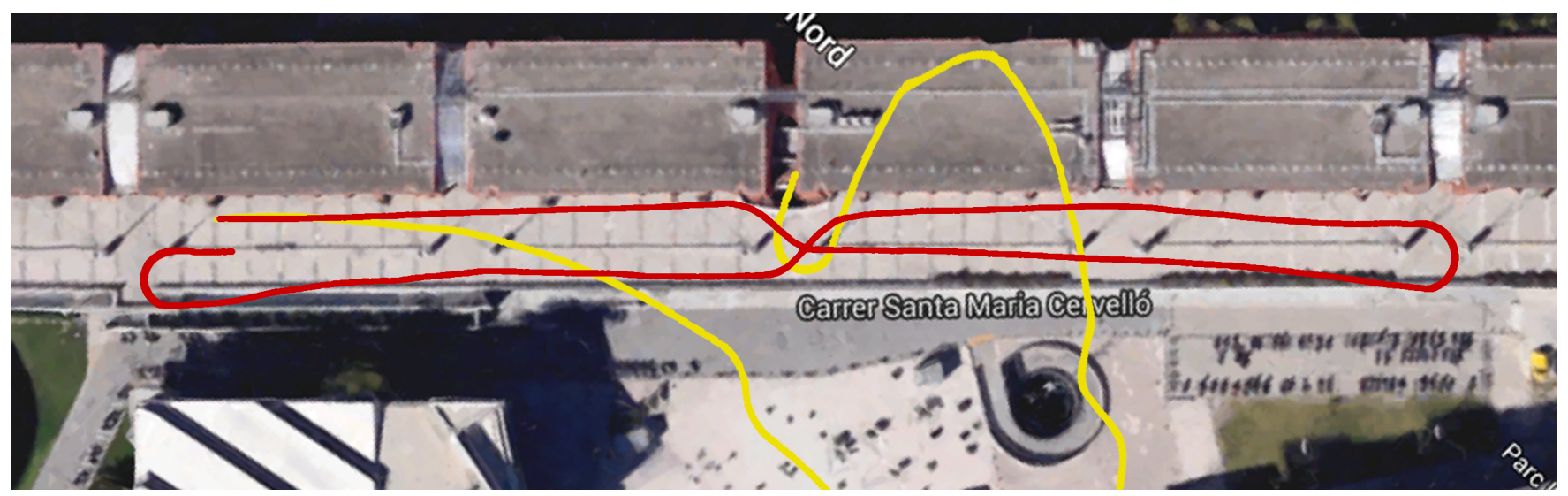

Fig. 8. Integrated pose for optimal sensor placement (CFG-A, red), and non-optimal one (CFG-B, yellow, only partial). Black dots show the path followed and black square departure point. Total length was about $350 \mathrm{~m}$. All paths plotted on a Google Maps picture of the experimental area.

velocity not included in the $2 \mathrm{D}$ twist state, but, however, adding a linear velocity component to the sensor devices, hence a Doppler velocity to radar detections. This happened in the middle of the third and fourth straight parts of the path. The reader can appreciate how two respective slight deviations on the vehicle's heading occurred is these points of the trajectory.

Such qualitative results illustrate that stereo radar odometry approach is a promising technique, providing accuracy at the level of a wheel encoder system, but able to observe the full 2D twist (lateral velocity also). Moreover, these results also demonstrates the relevance of sensor placement to achieve an accurate odometry estimate.

\section{CONCLUSIONS}

Stereo radar odometry, based on azimuth and Doppler data of radar detections, has been proposed recently by the automotive research community [8], [13], showing promising results. Following that approach, this paper contributes by providing a deep analysis on observability issues, as well as on optimal sensor placement. Initially, the paper presents a detailed formulation for the Doppler error function and its associated variance model. Thereafter, the paper analyses twist observability, showing theoretically why, at least, two radar devices are required. In the stereo radar case, degenerate situations are also identified, which impair observation of the vehicle twist, even when two sensors are used. The paper continues with a numerical analysis, proposing a methodology to find out which mounting pose configuration is the optimal one to estimate the twist. Finally, the paper illustrates with real world experiments the validity of such methodology, showing in a quantitative way that optimal configurations estimate better the vehicle twist than non-optimal ones. The paper also shows qualitative results after a trajectory of several hundreds meters.

In conclusion, stereo radar arises as a promising complementary odometry system, able to compute the full 2D twist of a vehicle, with accuracies at the level of other odometry systems. To estimate the full 2D twist $\left(v_{x}, v_{y}, \omega\right)$ is also of great interest for non-Ackerman platforms, as some industrial AGV's featuring lateral motion. Moreover, this approach completely avoids any data association step, neither with previous measurements, nor with any map landmark.

Outlier rejection techniques, sensor fusion with other odometry modalities, as well as integration in a SLAM framework, are among the future works we envisage.

\section{REFERENCES}

[1] M. Adams and E. Jose. Robotic navigation and mapping with radar. Boston: Artech House, 2012.

[2] S. Agarwal and K. Mierle et al. Ceres Solver. http://ceres-solver.org.

[3] M. Agrawal and K. Konolige. FrameSLAM: From Bundle Adjustment to Real-Time Visual Mapping. IEEE Transactions on Robotics, 24(5), October 2008.

[4] S. Amari and H. Nagaoka. Methods of Information Geometry. American Mathematical Soc., 2007.

[5] I. Baldwin and P. Newman. Road vehicle localization with 2d pushbroom lidar and $3 \mathrm{~d}$ priors. In Proc. IEEE International Conference on Robotics and Automation (ICRA), Minnesota, USA, May 2012.

[6] G. Brooker, D. Johnson, J. Underwood, J. Martinez, and L. Xuan. Using the Polarization of Millimeter-wave Radar as a Navigation Aid. Journal of Field Robotics, (32):3-19, 2015.

[7] H. Durrant-Whyte, D. Pagac, B. Rogers, M. Stevens, and G. Nelmes. An autonomous straddle carrier for movement of shipping containers. Robotics Automation Magazine, IEEE, 14(3):14 -23, 2007.

[8] D. Kellner, M. Barjenbruch, J. Klappstein, J. Dickmann, and K. Dietmayer. Instantaneous Ego-Motion Estimation using Multiple Doppler Radars. In Proceedings of the IEEE International Conference on Robotics and Automation (ICRA), Hong Kong, China. June, 2014.

[9] C. Lundquist, L. Hammarstrand, and F. Gustafsson. Road intensity based mapping using radar measurements with a probability hypothesis density filter. Signal Processing, IEEE Transactions on, 59(4):1397-1408, April 2011.

[10] T. Lupton and S. Sukkarieh. Visual-Inertial-Aided Navigation for High-Dynamic Motion in Built Environments Without Initial Conditions. IEEE Transactions on Robotics, 28(1), 2012.

[11] W. Maddern, G. Pascoe, and P. Newman. Leveraging Experience for Large-Scale LIDAR Localisation in Changing Cities. In Proceedings of the IEEE International Conference on Robotics and Automation (ICRA), Seattle, WA, USA, May 2015.

[12] D. Nistér, O. Naroditsky, and J. Bergen. Visual Odometry. In Proceedings of the IEEE Conference on Computer Vision and Pattern Recognition (CVPR), 2004.

[13] M Rapp, M. Barjenbruch, M. Hahn, J. Dickmann, and K. Dietmayer A Fast Ego-Motion Estimation Framework for Radar. In Proceedings of the European Conference on Mobile Robots, Lincoln, UK. 2015. 